\title{
Mix-Based Electronic Payments
}

\author{
Markus Jakobsson ${ }^{1}$ and David M'Raïhi ${ }^{2}$ \\ 1 Information Sciences Research Center, Bell Laboratories \\ Murray Hill, NJ 07974 \\ markusj@research.bell-labs.com \\ 2 Gemplus Corporation \\ 3 Lagoon Drive, Suite 300 \\ Redwood City, CA 94065-1566 \\ david.mraihi@gemplus.com
}

\begin{abstract}
We introduce a new payment architecture that limits the power of an attacker while providing the honest user with privacy. Our proposed method defends against all known attacks on the bank, implements revocable privacy, and results in an efficient scheme which is well-suited for smartcard-based payment schemes over the Internet.
\end{abstract}

\section{Introduction}

Since the conception of anonymous payment schemes by Chaum $\mathbf{r}$ in the early eighties, a lot of attention has been given to new schemes for transfer of funds. Anonymity and its drawbacks, in particular, has been a busy area of research lately, giving rise to many solutions for balancing the need for privacy against the protection against abuse. However, all of these schemes have been based on the same basic architecture as the pioneering scheme, which we suggest may be an unnecessary limitation. By rethinking the underlying privacy and fund-transfer architecture, we show that a stronger attack model can be adopted, and costs curbed.

It is well known that it never pays to make any link of a chain stronger than the weakest link. What has not been given much - if any - attention is that, in fact, it can be damaging to do so. The reason is that an attacker may selectively avoid using the weakest link components, thereby potentially enjoying protocol properties corresponding to the strongest link of the chain. More specifically, an attacker is implicitly given the option to use alternative tools in lieu of the given privacy-limiting portions of the scheme. For example, as appears to be the case for most Internet based payment schemes, the connection is the weakest link in terms of privacy, as a user reveals his IP address to some entity when establishing a connection. An attacker could physically mail in disks and ask to have the encrypted responses posted to newsgroups - and therefore successfully hide his IP address. An honest user would not be granted this same level of privacy: his address would only be concealed by means of an anonymizer, such as a mix-network (e.g., 5 I 2126.3 .3 ) or a crowd 27, both of which are based on the cooperation of some trusted participants. Therefore, if one link of the 
chain, corresponding to the privacy of one system component, is stronger than others, this potentially benefits an attacker without directly adding any value to the honest users.

We argue that it is unnecessary to employ a scheme with properties that can only be used by attackers. Moreover, by degrading the privacy of components until all components deliver the degree of privacy of the weakest link, efficiency improvements can be found. Thus, we can strengthen the defense against attacks (some of which might not be known to date) at the same time as we make the system less costly, without making the system less attractive to its honest users.

An example from the literature of this type of "double improvement" is the work of Jakobsson and Yung 171819 20, in which the bank robbery attack (in which the attacker obtains the secret keys of the banks) was introduced and prevented against, and the mechanisms employed to achieve this goal were used to improve the versatility and efficiency of the resulting scheme. Their scheme has two modes - one efficient off-line mode, for the common case, and one rather costly on-line mode, to be used only after a successful bank robbery. By making sure that an attacker can never enjoy a higher degree of privacy than an honest user, and implementing mechanisms for a quorum of servers to selectively revoke privacy, we obtain the same degree of security as the on-line mode of the scheme by Jakobsson and Yung, at a cost that is comparable to the off-line mode of the same scheme.

Our scheme is, unlike other payment schemes with privacy, not based on blind signatures (or variations thereof), but its privacy is derived solely from the use of a mix-network. It does not allow an attacker any higher degree of privacy than honest users, but all users enjoy the following degree of privacy: As long as no quorum of bank servers is corrupted or decides to perform tracings, and no payer or payee reveals how they paid or were paid, the bank servers can only learn the number of payments to and from each account during each time period (e.g., one month.) Moreover, as is appropriate, the transactions are independent in the sense that knowledge about one transaction gives no knowledge of another transaction (other than reducing the number of remaining possibilities.)

We note that if one bank server and one merchant collude, then they can together remove the privacy for transactions that this merchant was involved ir 1 Whereas this is a lower degree of protection than what many other schemes offer, it makes sense in a setting where users are not concerned with the bank potentially learning about a few of their transactions, as long as the majority of the transactions are secret. We believe that this is an adequate degree of protection to avoid profiling of users performing every-day transactions, which is a degree of privacy that society seems to agree on should be granted.

The communication and computational costs for the different transactions are very similar to those of other schemes, but the amount of data stored by payers and merchants is significantly lower, making our suggested scheme particularly suitable for smartcard implementations.

${ }^{1}$ This limitation can be avoided at the cost of a lower propagation rate, as will be discussed onwards. 


\section{Using Smartcards for Payment Schemes}

Let us for a moment take a step back from what features are desirable in a payment scheme, and focus on what is absolutely necessary. In order not to have to rely on any physical assumption, we need some form of authentication and encryption for security and privacy. Also, in order for the user not to have to put excessive trust in other entities, public key solutions have to be used. Therefore, let us call signature generation and encryption the minimal requirements (where the user performs all or some of the computation involved in performing the transactions.)

The most suitable and least expensive way of meeting these requirements in an environment with portable payment devices would be to develop and use a very simple smartcard with a hardware accelerator for modular multiplications, and with a minimum of memory (since what is expensive in the design of smartcards is EEPROM and RAM). Such a product would be perfect for generating and verifying signatures (e.g. DSA 25 or RSA 28 signatures), and the use of a co-processor would allow very fast public-key certificate verification. It would offer the necessary operations required by all e-commerce applications. However, the use of such a smart card desperately clashes with existing solutions for anonymous e-commerce, since these require high amounts of storage, complicated protocols, and in many cases rely to some extent on tamper-resistance. (Note, however, that there are numerous payment schemes that are smart-card based, e.g., 1023 . This is, in fact, a case in point, since these schemes either require expensive special-purpose smart cards, or limit the safe usage of the payment scheme due to its reduced security.)

The main advantage of our scheme is that we overcome the major drawbacks related to the original e-coin paradigm: using a mix-decryption scheme to provide controlled anonymity, we build a counter-based scheme where the participants do not need to store anything but their secret keys and a small amount of userrelated information. In our setting, all participants could therefore be represented as owners of an inexpensive and simple smart-card of the type discussed above. We demonstrate how to build a payment scheme offering privacy, using only features of such a minimalistic cryptographic smartcard. Our solution offers:

- flexibility and plug-and-play ability to users: the same device enables homebanking, e-commerce and potentially extensions to applications such as privacy-enhanced email,

- strong protection to users, issuers and governments by the use of controlled and balanced privacy.

\section{Intuitive Approach}

Instead of using the common token approach, we will use an account-based approach, where a payer requests to have a payment made from his account to another account. The payer's identity will be known to the bank, who will deduct the appropriate amount from his account after a valid, authenticated 
payment request has been received. However, the merchant's identity will not be obvious from this request. More specifically, it will be encrypted using a public key, whose corresponding secret key is distributively held by a set of servers, which are controlled by banks and government entities. At given time intervals, or after a certain number of payment orders have been received, these will be collectively decrypted by a set of servers holding secret key shares. This is done in a manner that does not reveal the relationship between any given encrypted payment order and its corresponding cleartext payment order, the latter which contains the account number of the merchant to be paid. After the collective decryption of all encrypted payment orders, all the accounts indicated by these will be credited appropriately.

In order to increase the propagation speed between initiation of payment (the submission of the encrypted payment order) and the acceptance of a payment by the merchant, we can let the payer prove to the merchant that the payment order has been accepted by the bank, and that the eventual decryption of the payment order will result in the crediting of the merchant's account being made.

We note that in either case, there is no need for the merchant to be in direct contact with the bank (there is no deposit), and neither the payer nor the merchant needs to store a large amount of information, but just a sufficient amount to generate vs. verify payment orders. The main computation will be done by the much more powerful bank servers, and without any strong online or efficiency requirements being imposed.

\section{Related Work}

When the concept of electronic payments was introduced (see $7 \mathrm{~g}$ ) there was a strong focus on perfect privacy. Lately, as possible government policies have started to be considered (e.g., 34), and attacks exploiting user privacy have been discovered (e.g., $3 \mid$ I7), the attention has shifted towards schemes with revocable privacy.

In ?, Brickell, Gemmell and Kravitz introduced the notion of trustee-based tracing, and demonstrated a payment scheme that implemented computational privacy that could be revoked by cooperation between the bank and a trustee. The concept of fair blind signatures was independently introduced by Stadler, Piveteau and Camenisch $3 . \%$; this is a blinded signature for which the blinding can be removed by the cooperation between the signer (the bank) and a trustee. This type of signatures were employed in payment schemes in 54 , and a smartcard based variation was suggested in $\% 4$. In $1 \% \mathrm{II}$, methods were introduced allowing the trustee not to have to be on-line during the signature generation, by employing so called indirect discourse proofs, in which the withdrawer proves to the bank that the trustee will later be able to trace.

In the above schemes, a cooperating bank and trustee were able to trace all properly withdrawn coins, but not coins obtained in other ways. The underlying attack model was strengthened by Jakobsson and Yung 17 , by the introduction of the bank robbery attack, in which an attacker compromises the secret keys 
used for signing and tracing, or forces the signers to produce signatures using an alternate generation protocol; their proposed solution protects against this attack and permits all coins to be traced, and any coin to be successfully blacklisted after a short propagation delay. Also, the degree of trust needed was decreased, by the use of methods to assure that the tracing and signing parties do not cheat each other. This work was improved on in 181920 by the distribution of the parties and the introduction of a minimalistic method for privacy revocation (i.e., verifying if a given payment corresponds to a given withdrawal.)

We achieve the same protection against attacks on the bank as the above constructions, and additionally, by shifting the model from a coin-based to an account-based model, make bank robberies futile: If the secret keys of the banks should be compromised, this only allows the attacker to trace payments, and not to mint money. Additionally, we meet the level of functionality introduced in 1819 in terms of methods for tracing, and distribution of trust and functionality. Finally, by the shift in architecture, we remove the propagation delay for blacklisting - in fact, we avoid having to send out blacklists to merchants altogether.

However, our scheme requires the payer to be able to communicate with a bank or clearing center for each payment. Also, it does not offer the same granularity of payments that the use of challenge semantics in 1718 could, but potentially requires multiple payments to match a particular amount (much like for many coin based schemes).

On the other hand, we are able to reduce the amount of information stored by the payer (who only has to store his secret key.) Moreover, the merchants neither have to store any payment-related information (after having verified its correctness), nor do they ever have to connect to the bank. (In some sense, one might say that the deposit is performed by the payer.) This allows for a very efficient implementation in a smartcard based Internet setting (where the smartcards are used by the payers to guarantee that only authorized users can obtain access to funds.)

The anonymity in our scheme is based solely on the use of a mix-network, a primitive introduced by Chaum 6 . We demonstrate an implementation based on a general mix-network (e.g., 6 3.3 ), and state and prove protocol properties. We then consider the use of a particular, recently proposed type of mix-network I 2126 , and discuss how its added features can further strengthen the payment scheme. The result is an efficient and practical payment scheme, well suited for Internet implementation, that prevents by all known attacks by ensuring that an attacker never can obtain any more privacy than an honest user is offered. The privacy is controlled by a conglomerate of banks and ombudsmen, a quorum of which have to cooperate in order to revoke it.

Another scheme that uses a mix-network (or some other primitive implementing anonymous communication) is the payment scheme of Simon 30. That scheme is similar to ours also in that it takes the "minimalist approach" as well, building an anonymous payment scheme from a small set of simple components, and in that the account information to some extent is kept by the bank. More 
specifically, Simon's scheme uses a mix-network to communicate preimages to function values that are registered as having a value. When the merchant receives such a payment, he deposits it by sending (again, using the mix-network) the preimage along with a new function value, to which only the merchant knows the preimage. The bank cancels the old function value and associates value with the new function value. Whereas this allows linking of payments through a chain, it still offers a high degree of privacy to its users. Although Simon's scheme is not directly concerned with limiting the privacy an attacker can enjoy, and there are several privacy-related attacks that can be performed (since there is no direct notion of revocation of privacy), it still appears to be the case that the scheme is secure against some of the strongest attacks (e.g., bank robbery.) This is due to the fact that the representation of value is effectively controlled by the bank by the storage of data by the bank, and not solely linked to having a bank signature.

An interesting observation is the close relationship between our proposed payment scheme and election schemes. More specifically, it can be seen that our payment scheme can be used as an election scheme with only minor modifications; the resulting election schem ${ }^{2}$ implements most recently proposed features, and allows for multi-bit votes to be cast.

\section{Model}

Participants: There are five types of participants, all modeled by polynomialtime Turing machines: payers, merchants, bank $\mathbf{3}$ a transaction center, and the certification authority. The payers and merchants have accounts with banks (but not necessarily the same banks); the transaction center processes transfers between accounts of payers and merchants, and is controlled by a conglomerate of banks; the certification authority issues certificates on all other participants' public keys, and may be controlled by banks and government organizations. The transaction center knows for each payer's public key the identity of the payer's bank. (If a user has several banks, he correspondingly has several public keys, one per bank.)

Trust: The payers and merchants trust that the banks will not steal their money. The payers trust for the privacy of a particular transaction that the merchant of the transaction does not collude with bank servers or otherwise attempt to reveal their identities. The payers trust for their general privacy that there is not a quorum of dishonest, cooperating banks constituting a corrupt transaction center.

Computation: We base our general scheme on the existence of a mix-network. Such a scheme can be produced based on any one-way function. We also

\footnotetext{
${ }^{2}$ Instead of encrypting the account number of the payee, the payer/voter would encrypt his vote.

${ }^{3}$ It is possible to substitute some bank servers for ombudsman servers, whose aim it is to limit illegal tracing transactions by bank servers. These will not have to be trusted with funds, and only hold tracing keys. Due to a shortage of space, we do not elaborate on how exactly to implement these.
} 
present a scheme using a recently proposed type of ElGamal based mixnetwork 12126 .

\section{Requirements}

We will require the following from our scheme:

Unforgeability: It is not possible for a coalition of participants not including a quorum of bank servers to perform valid payments for a value $v$ exceeding the value charged to these participants.

Impersonation safety: It is not possible for a coalition of cheating participants to perform a transaction resulting in an honest participant being charged more than what he spent.

Overspending blocking: It is not possible for a coalition of participants to perform payments that are accepted by merchants as valid, for an amount exceeding their combined limits, or exceeding what they will be charged for.

Payment blocking: It is always possible for a user to block all payments from his account, going into effect immediately.

Revocability: The bank of any user can restrict the rights of the user to perform payments. If a user has no bank agreeing to a payment, the payment will not be performed.

Framing-freeness: It is not possible for a coalition of participants not including an honest user to produce a transcript corresponding to a payment order from the honest user.

Uniform anonymity: The probability for any coalition of participants, not including a quorum of bank (and ombudsman) servers, to determine from whom a payment was made and to whom (assuming that neither of these parties collaborate with the same servers) is non-negligible better than a guess, uniformly at random from all possible pairs, given all pairs of payers and merchants corresponding to the input and output of the mix-network. It is not possible for a coalition of participants to obtain a higher degree of anonymity by forcing alternative protocols to be used.

Traceability: Any quorum of bank server 1 are able to perform the following actions:

1. Given identifying information about a payer (and possibly a particular payment transaction of the payer), establish the identity of the receiver of the corresponding payment(s).

2. Given identifying information about a merchant (and possibly a particular payment transaction to the merchant), establish the identities of the corresponding payer(s).

3. Given identifying information about a payer; a particular payment transaction from the same; a merchant; and a particular payment transaction to the same, establish whether these correspond to each other.

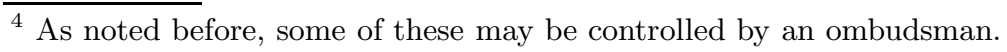




\section{Quick review: Mix-networks}

Before presenting our scheme, let us briefly review what a mix-network is: In general terms, it is a set of servers that serially decrypt and permute lists of incoming encrypted messages. Here, the messages are either encrypted using all the individual public keys of the servers, or using one public key, where the corresponding secret key is shared by the mix servers.

The scheme implements privacy as long as at least one of the active mix servers does not reveal what random permutation he applied, and the encryption scheme is probabilistic, so that it is not possible to compute the same encrypted messages that constituted the input given the decrypted messages that constitute the output.

Some of the schemes introduced are robust, meaning that it is not possible for some subset of cheating servers participating in the mix-decryption to make the final output incorrect (without this being detected by the honest participating servers.)

We present a more detailed explanation of one particularly useful class of mix-networks in section 9

\section{Paying and Tracing (General Version)}

\subsection{Setup}

For each time interval between accounting sessions (in which the payment orders get decrypted and the merchants credited) a new public key may used (we will elaborate on when this is necessary onwards.) These public keys can be broadcast beforehand. The corresponding secret keys are known only to the transaction center, and are kept in a distributed manner so that any quorum of mix-servers can calculate it.

Each party is associated with a public key, which is registered with the transaction center if the party in question is authorized to perform payments. Only this party knows the corresponding secret key, which is used to sign payment orders and possibly other data which needs to be authenticated. The signature scheme employed for this is assumed to be existentially unforgeable.

\subsection{Paying}

There are three phases of the payment scheme: negotiation, initiation of payment, and completion of payment. In the first, the payer and the merchant produce a description of the transaction; in the second, the transaction is being committed to and the payer's account debited; and in the third, the merchant's account is credited accordingly. Note that the transaction becomes binding in the second phase already, so the transfer of the merchandise bought can be initiated right after the second phase has finished, and the third phase may be performed at a later point. This is a scenario rather similar to the credit card scenario, in which the payer commits to the transfer much before the merchant receives the funds. 


\section{Negotiation}

In the negotiation phase, the payer and the merchant agree on an exchange, i.e., a description of the merchandise, how it will be delivered, the price of the merchandise, etc. We let $m$ be a hashed-down description of this contract. Furthermore, the merchant gives the payer his account number $a$ (which specifies the bank as well as identifies the merchants account in this bank) and a serial number $s$ for this transaction. If the payment will constitute of several coins, a suite $s_{1}, \ldots, s_{k}$ of such serial numbers is given, each one representing one partial payment. A payment order $o$ is of the form $m|a| s$. (If a suite of serial numbers is used, then a corresponding suite of payments orders $o_{1}, \ldots, o_{k}$ will be generated.)

\section{Initiation of Payment}

(a) The payer encrypts a batch of payment orders $o_{1}, \ldots, o_{n}$ using the public key encryption scheme of the transaction center (which corresponds to the mix-network). These payment orders do not have to correspond to one transaction only, or have the same denomination, but may stem from multiple simultaneous transactions and be of different values. The result is a batch of encrypted payment orders, $\tilde{o}_{1}, \ldots, \tilde{o}_{n}$. If different denominations are supported in the system, a description $d$ is appended, describing what the values of the different transactions are. If only one denomination is supported, $d$ is the empty string. The payer signs $\tilde{o}_{1}, \ldots, \tilde{o}_{n}, d$ using his private key. The resulting signature is $\sigma$. The payer sends $\tilde{o}_{1}, \ldots, \tilde{o}_{n}, d, \sigma$ to the transaction center.

(b) The transaction center verifies the signature $\sigma$. One of two approaches is taken in order to avoid replay attacks: either, the payer needs to prove knowledge of some part of the posted message in a way that depends on time, or the transaction center keeps the posted messages in a sorted list and ignores any repeated posting. If the latter approach is taken, the payment orders are encrypted using a new public key for each payment period (the time in between two accounting phases.)

The transaction center then may verify that the payer has sufficient funds. This may be required of certain banks, or for amounts above some threshold; alternatively, verifications may be performed randomly or in batches to lower the processing times and costs.

Each valid encrypted payment order $\tilde{o}_{i}$ is added to an internal list of payments to be performed; there is one such list for each denomination. The payers' accounts are debited accordingly, either immediately or in batches. The transaction center signs each individual valid encrypted payment order $\tilde{o}_{i}$, resulting in a signature $\sigma_{i}$. The transaction center uses different public keys for different denominations, or appends a description of the denomination before signing. It sends $\sigma_{1}, \ldots, \sigma_{n}$ to the payer.

${ }^{5}$ Suites of transactions orders corresponding to the same transaction may be signed together to improve efficiency. 
(c) The payer sends a suite of signed, encrypted payment orders (of the format $\left.\tilde{o}_{i}, \sigma_{i}\right)$ to the corresponding merchant. He also sends a proof of what the encrypted messages are (in some cases, this amounts plainly to show the plaintext.)

(d) The merchant verifies that $\sigma_{i}$ is the transaction center's signature on the payment order $\tilde{o}_{i}$, and that the decrypted value $o_{i}$ is of the form $m|a| s$ for valid a valid merchandise description $m$, the merchant's account number $a$, and a sequence number $s$ not yet received.

If the above verification goes through, then the merchant stores the sequence number, and delivers the merchandise purchased.

\section{Completion of Payment}

At given intervals, the transaction center decrypts all the payment orders using the mix-decryption scheme employed. The result is a plaintext list of payment orders for each denomination. The items of these lists indicate what accounts are to be credited, and by how much. The banks corresponding to the accounts indicated credit these accounts accordingly.

Remark 1: If steps $2 \mathrm{c}$ and $2 \mathrm{~d}$ are excluded, we can avoid the privacy assumption that the merchant is not colluding with a bank server. The cost for this is a lower propagation speed, i.e., the purchase will not be possible to complete until the bank servers has decrypted the payment order batch.

Remark 2: Note that the payment orders can be generated by the payers without any real interaction between the payer and the merchant. This is possible if $m$ is a publicly available description (e.g., the hash of an advertisement sent out by the merchant,) $a$ is publicly available as well, and $s_{1}, \ldots, s_{k}$ is selected uniformly at random from a space that is large enough to avoid collisions.

Remark 3: In order to limit the amount of storage needed for the merchant, the sequence numbers may contain a date and time stamp, and will only be accepted - and stored - within an interval associated with this timestamp.

\subsection{Tracing}

If a general mix-network is employed, only the two basic tracing operations can be performed efficiently, tracing from a payer to a payment order, and from a payment order to a payer. We will later look at how this can be extended (and simplified) for an ElGamal based mix-network.

\section{Payer $\rightarrow$ Payment Order}

The trace is performed simply by decrypting the encrypted payment order $\tilde{o}$ in question, arriving at the plaintext payment order $o$, which specifies the account to which the payment is performed.

\section{Payment Order $\rightarrow$ Payer}

The trace is performed as follows: the mix-servers reverse the computation of $o$ from $\tilde{o}$ step by step, proving to each other that each step is valid. Depending on what type of mix-network is employed, it may be necessary to 
keep intermediary values, such as partial decryptions and the permutations employed; if these are not available, then they can be re-generated by decrypting the entire bulletin board again.

\section{$9 \quad$ Paying and Tracing (ElGamal Version)}

If the recently proposed type of mix-decryption scheme (different methods independently proposed by Abe II, by Jakobsson 2I, and by Ogata, Kurosawa, Sako, and Takatani 20 ) is used by the transaction center, this allows several improvements to be made. Let us first briefly describe this mix-decryption scheme, and then elaborate on the advantageous consequences of using it.

1. The input to the scheme is a list of ElGamal encrypted messages (encrypted with the public key of the transaction center, i.e., mix center.) The output is a permuted list of the corresponding decrypted values.

2 . The decryption process can be performed by any $k$ out of $n$ servers, who share the secret key for decryption. The participants with whom the encryptions originated need not be involved.

3. No subset of less than $k$ out of $n$ mix servers can perform the decryption.

4. The decryption process is robust, meaning that if any server(s) should cheat, then the cheating will be detected, and their identities become known to the remaining servers.

5. No subset of cheating servers can correlate items of the input to items of the output (unless they already know the corresponding plaintext messages.)

6. The decryption process is efficient.

If this scheme is employed, the following holds:

1. As long as there exists a quorum of honest mix-servers (controlled by the banks), the decryption process will be possible 9 .

2. As long as at least one of the participating mix-servers is honest, the correctness of the output is guaranteed 7

3. As long as there is no dishonest quorum of mix-servers, the privacy of users will be guaranteed.

The tracing scheme can be simplified as well, and makes it unnecessary for the banks to store the contents of the bulletin board, or intermediary computation, in order to perform traces. The following three types of tracing can be performed:

\section{Payer $\rightarrow$ Payment Order}

The trace is performed simply by decrypting the encrypted payment order $\tilde{o}$ in question, arriving at the plaintext payment order $o$, which specifies the account to which the payment is performed.

${ }^{6}$ This is an important issue, since otherwise it would be possible for one corrupt bank or ombudsman (controlling one mix-server) to stop all payments in one payment period (unless the payers volunteer to repeat their payments!)

7 This is important, or a subset of mix-servers could manipulate the payments without being detected. 


\section{Payment Order $\rightarrow$ Payer}

Here, the given decrypted payment order is to be compared to the encrypted input items. When a match is found, the corresponding payer identifier is output. This can be done by first blinding both the payment order and all the input item, 8 using the same distributively held blinding factor, and then decrypt all the blinded input items, after which a match is found. If the mix-decryption scheme by Jakobsson 21 is used, then the trace can be performed by computing a tag, corresponding to the input to the mixnetwork, and comparing this tag to the partially decrypted (but still blinded) encrypted payment orders. We refer to 2.1 for a more detailed description.

3. (Payment Order, Payer $) \rightarrow$ (yes/no)

This trace is performed by computing the above tag from the encrypted payment order in question, and verify whether this tag corresponds to the decrypted payment order (without revealing any other information than this one bit.) This can be done using the verification protocol for undeniable signatures $x$, or using a small modification of the first method described above.

\section{Claims}

The basic scheme satisfies the following requirements (for a quorum size of all participating bank servers, who are assumed to follow the protocol, although they may be curious to learn additional information): unforgeability, impersonation safety, overspending blocking, payment blocking / revocability, uniform anonymity, and framing-freeness. Additionally, the two first modes of tracing detailed in traceability are possible.

The ElGamal based scheme satisfies (without any condition on quorum size or behavior of non-cooperating servers) the above requirements, and full traceability. We prove these claims in the Appendix.

\section{Performance Analysis}

This section demonstrates that our proposed scheme is sufficiently efficient to be practical in a mobile setting, in which the user's device is a portable token, such as a smart-card with restricted capacities in terms of computational power and memory storage.

Although our solution is also competitive in a PC-based setting, it is the mobile setting that is the most restrictive. We note also that whereas a good performance w.r.t. the mix-decryption is important, it is not vital for the efficiency of the scheme, since the mix-decryption is performed off-line and in batches, and does not involve the payer or merchant, nor requires these to wait for the result of

\footnotetext{
${ }^{8}$ An ElGamal encryption $(a, b)$ can be blinded using a blinding factor $\delta$ by computing $\left(a^{\delta}, b^{\delta}\right)$. This results in a blinding of the plaintext message $m$ to $m^{\delta}$, but in encrypted form.
} 
the operation. In fact, the employment of the mix-network will be transparent to the users in the case where a threshold-based scheme (such as ElGamal) is used. (For a treatment of the efficiency of the mix-decryption schemes in question, we refer to the corresponding papers where these are treated in detail.)

Table 1 summarizes computational performance of two smartcards with cryptographic accelerators: Smartcard the new smartcard product from Siemens 29, used for instance in the Gemplus GPK range, and the Hitachi 16 enhanced chip for smartcards. Performance of the Siemens SLE66X160S has been carefully evaluated, running routines on emulator at a $3.68 \mathrm{MHz}$ clock frequency and with the worst-case set of parameters (all the exponent bits set).

\begin{tabular}{l|ccc} 
Data Length $l$ (bits) & 512 & 768 & 1024 \\
\hline H8/3113 & $90 \mathrm{~ms}$ & $350 \mathrm{~ms}$ & $700 \mathrm{~ms}$ \\
SLE66CX160S & $186 \mathrm{~ms}$ & $593 \mathrm{~ms}$ & $1045 \mathrm{~ms}$
\end{tabular}

Table 1. - Exponentiation Timings for exponent $=1_{l}$

Let us now consider a full implementation of the scheme. The smartcard used is based on the Siemens chip (GPK card) giving us timings around $300 \mathrm{~ms}$ for the 1024-bit RSA signature (with CRT) and $200 \mathrm{~ms}$ for the El-Gamal encryption. The total time expected for processing a payment order is approximately the time required for encrypting and signing data: The communication time for processing such transactions is around $100 \mathrm{~ms}$ when transmitted according to 7816-4 standard at $115,200 \mathrm{bd}$. Since the user does not need to update a transaction log, writing to EEPROM (which is quite time consuming) is avoided.

We performed various practical tests confirming that the total time for issuing a payment order should be around 1 second, as detailed in table 2 We assume that:

1. the time spent for checking at merchant and center is negligible (we consider a protocol overhead of $100 \mathrm{~ms}$ )

2. communication is performed at $115,200 \mathrm{bds}$

3. 512-bit modulus for ElGamal and 1024-bit modulus for RSA are practical and secure parameter sizes

\section{Acknowledgments}

We want to thank Moti Yung and David Kravitz for interesting discussions before resp. during the conception and writing of the article.

9 Timings are estimated since the chip has not yet been introduced, but is expected to be released during 1998 . 


\begin{tabular}{|l||c|}
\hline Operation & Processing Time \\
\hline 512-bit El-Gamal Encryption & $227 \mathrm{~ms}$ \\
Hashing Ciphertext (SHA) & $32 \mathrm{~ms}$ \\
1024-bit RSA Signature & $289 \mathrm{~ms}$ \\
Sending to center & $110 \mathrm{~ms}$ \\
Sending to merchant & $110 \mathrm{~ms}$ \\
Checking Overhead & $100 \mathrm{~ms}$ \\
\hline Total & $868 \mathrm{~ms}$ \\
\hline
\end{tabular}

Table 2. Transaction Time Evaluation

\section{References}

1. M. Abe, "Universally Verifiable Mix-net with Verification Work Independent of the Number of Mix-centers," Eurocrypt '98.

2. E. Brickell, P. Gemmell, D. Kravitz, "Trustee-based Tracing Extensions to Anonymous Cash and the Making of Anonymous Change," Proc. 6th Annual ACMSIAM Symposium on Discrete Algorithms (SODA), 1995, pp. 457-466.

3. H. Bürk, A. Pfitzmann, "Digital Payment Systems Enabling Security and Unobservability," Computers and Security 8/5 (1989), pp. 399-416

4. J. Camenisch, U. Maurer, M. Stadler, "Digital Payment Systems with Passive Anonymity-Revoking Trustees," Computer Security - ESORICS 96, volume 1146, pp. 33-43.

5. J. Camenisch, J-M. Piveteau, M. Stadler, "An Efficient Fair Payment System," 3rd ACM Conf. on Comp. and Comm. Security, 1996, pp. 88-94.

6. D. Chaum, "Untraceable electronic mail, return addresses, and digital pseudonyms," Communications of the ACM, ACM 1981, pp. 84-88

7. D. Chaum, "Blind Signatures for Untraceable Payments," Advances in Cryptology - Proceedings of Crypto '82, 1983, pp. 199-203.

8. D. Chaum, H. Van Antwerpen, "Undeniable Signatures," Crypto '89, pp. 212-216

9. D. Chaum, A. Fiat and M. Naor, "Untraceable Electronic Cash," Advances in Cryptology - Proceedings of Crypto '88, pp. 319-327.

10. Digicash, see www.digicash.com

11. G.I. Davida, Y. Frankel, Y. Tsiounis, and M. Yung, "Anonymity Control in ECash Systems," Financial Cryptography 97.

12. Y. Frankel, Y. Tsiounis, and M. Yung, "Indirect Discourse Proofs: Achieving Efficient Fair Off-Line E-Cash," Advances in Cryptology - Proceedings of Asiacrypt 96, pp. 286-300.

13. M. Franklin and M. Yung, "Towards Provably Secure Efficient Electronic Cash," Columbia Univ. Dept of C.S. TR CUCS-018-92, April 24, 1992. (Also in Icalp-93, July 93, Lund Sweden, LNCS Springer Verlag).

14. M. Franklin and M. Yung, "Blind Weak Signatures and its Applications: Putting Non-Cryptographic Secure Computation to Work," Advances in Cryptology Proceedings of Eurocrypt '94

15. Gemplus, "Gemplus Public Key (GPK) Cards", October 1996.

16. Hitachi, "Product directory: H8 Range", 1997.

17. M. Jakobsson and M. Yung, "Revocable and Versatile Electronic Money," 3rd ACM Conference on Comp. and Comm. Security, 1996, pp. 76-87. 
18. M. Jakobsson and M. Yung, "Applying Anti-Trust Policies to Increase Trust in a Versatile E-Money System," Financial Cryptography '97.

19. M. Jakobsson and M. Yung, "Distributed 'Magic Ink' Signatures," Eurocrypt '97, pp. $450-464$

20. M. Jakobsson, "Privacy vs. Authenticity," PhD Thesis, University of California, San Diego, Department of Computer Science and Engineering, 1997. Available at http://www-cse.ucsd.edu/users/markus/.

21. M. Jakobsson, "A Practical Mix," Eurocrypt '98, available at www . bell-labs.com/user/markusj/

22. S. Low, N. Maxemchuk, "Anonymous Credit Cards," 2nd ACM Conference on Computer and Communications Security, 1994, pp. 108-117.

23. Mondex, see www.mondex.com

24. D. M'Raïhi, "Cost-Effective Payment Schemes with Privacy Regulation," Advances in Cryptology - Proceedings of Asiacrypt '96.

25. National Institute for Standards and Technology, "Digital Signature Standard (DSS)," Federal Register Vol 56(169), Aug 30, 1991.

26. W. Ogata, K. Kurosawa, K. Sako, K. Takatani, "Fault Tolerant Anonymous Channel," Information and Communications Security, '97, pp. 440 - 444

27. M. Reiter, A. Rubin, "Crowds: Anonymity for Web Transactions," ACM Transactions on Information and System Security, April, 1998.

28. R. Rivest, A. Shamir and L. Adleman, "A method for Obtaining Digital Signatures and Public-Key Cryptosystems", Communications of the ACM, v. 21, n. 2, Feb 1978, pp. 120-126.

29. Siemens, "ICs for Chip Cards", Customer Information, June 1997.

30. D. Simon, "Anonymous Communication and Anonymous Cash," Crypto '96, pp. 61-73.

31. S. von Solms and D. Naccache, "On Blind Signatures and Perfect Crimes," Computers and Security, 11 (1992) pp. 581-583.

32. M. Stadler, J-M. Piveteau, J. Camenisch, "Fair Blind Signatures," Advances in Cryptology - Proceedings of Eurocrypt '95, 1995.

33. P. Syverson, D. Goldschlag, M. Reed, "Anonymous connections and onion routing," IEEE Symposium on Security and Privacy, 1997.

34. B. Witter, "The Dark Side of Digital Cash," Legal Times, January 30, 1995

\section{Appendix: Proofs of Claims}

We outline the proofs of the claims stated in section 10. where applicable, the proofs have one part relating to the general scheme (assuming that all mix servers of the mix-network used cooperate) and one to the ElGamal scheme (assuming a quorum of specified size to cooperate). The final version will include full proofs, which are kept short here due to space shortage.

Theorem 1: The schemes implement unforgeability.

Proof of Theorem 1: (Sketch)

First, in order to post a payment order that will be accepted, a valid signature of the payer has to be produced; by the soundness of the underlying existentially unforgeable signature scheme, this can only be done by the account owner in question. Second, in order for a party to be credited, it is necessary that one 
of the the output items of the mix-network specifies their account number. If a general mix-network is used, the mix-servers are assumed to perform the valid decryption (if they do not, they can only alter who gets credited, and not the number of payments performed); if the suggested ElGamal mix-network is used, then by the robustness of this, the outputs are guaranteed to be valid if at least one participating mix server is honest. Therefore, only the participating payers will be charged, and only the intended payees debited. The amount of the credits cannot exceed the amount of debits.

Theorem 2: The schemes implement impersonation safety.

\section{Proof of Theorem 2:}

By the soundness requirements of the identification schemes used to obtain access to accounts, only authorized parties are going to get access to their accounts, as long as they keep their secret keys secret. Also, it is not possible for an adversary to produce a signature by a payer not cooperating with him. This follows from the assumption that the signature scheme used by the payer to sign an encrypted payment order is existentially unforgeable: a new signature cannot be produced without knowledge of the secret key. If the same signed message is reposted in the same payment period, then it will be removed, either after the payer has failed to prove knowledge of some part of the post, or since duplicates are ignored (depending on the approach taken.) Since we, for the approach where duplicates are removed, require the payment orders to be encrypted using a public key specific to the time period between two accounting sessions (corresponding to the mix-decryptions), and the relationship between the secret keys of intervals is unknown, it is not possible to force an old encryption to be accepted in a new time interval.

Theorem 3: The schemes implement overspending blocking.

This follows trivially from Theorem 1, and the fact that for each payment one signature has to be generated, and for each such signature, one account is billed.

Theorem 4: The schemes implement payment blocking / revocability.

\section{Proof of Theorem 4: (Sketch)}

Since a payment can only be made from an account by producing a signature, and sending this to the transaction center, and the signatures used for this will identify the account owners, it is possible for an account owner to stop all payments from his account by requesting that no signature of his is accepted by the transaction center. This corresponds to either putting his public key on a blacklist, or by removing it from the list of valid public keys. Similarly, the same can be done by the bank of an account holder to block the account holder access to his funds.

Theorem 5: The schemes implement framing-freeness.

Proof of Theorem 5: (Sketch)

This follows from Theorem 2, and the fact that nobody but a user (including the bank servers) knows the secret key of this user. Therefore, it is not possible to 
produce a set of transcripts indicating that a party performed a given payment, without the cooperation from this party.

Theorem 6: The schemes implement uniform anonymity.

Proof of Theorem 6: (Sketch)

The only way a payment can be initiated is by posting an encrypted and signed payment order on the bulletin board of the transaction center. The signature identifies the payer; if the identity of the signer cannot be established (or he is not authorized to make payments,) then the posted message will be ignored. Then, the only way that merchants can be credited is by decrypting all the payment orders. The link between encrypted an decrypted items can only be established by a quorum of mix-servers. If fewer than this could correlate the input and output items, this contradicts the assumption that the ElGamal encryption scheme is probabilistic. By the traceability option (see next theorem), the anonymity of any valid encrypted or decrypted payment order can be removed, and therefore, all participants enjoy the same degree of anonymity.

Theorem 7: The general scheme implements the two first methods for traceability; the ElGamal based scheme implements full traceability.

\section{Proof of Theorem 7: (Sketch)}

We have established above that for each account that is credited, the transaction center has a signature of the party whose account will be debited: the link between the two can always be established by either decrypting a single posted encrypted and signed payment order (tracing from payer to payment order), or by (potentially partially) re-encrypting a decrypted payment order (arriving at the payer information from the payment order.) In addition, the third tracing option (comparison) can be performed in the ElGamal based scheme, by the use of the verification protocol for undeniable signatures. 